\title{
The Art of Hormone Measurements with Emphasis on Specificity Resulting in Diagnostic and Management Improvements
}

\author{
Kanegusuku AG ${ }^{1 *}$ and Soldin SJ ${ }^{1,2 *}$ \\ ${ }^{1}$ Department of Laboratory Medicine, National Institutes \\ of Health, USA \\ ${ }^{2}$ Department of Medicine, Georgetown University, USA \\ \#Authors Contributed Equally \\ *Corresponding author: Steven J. Soldin, \\ Department of Laboratory Medicine, Clinical Center, \\ National Institutes of Health, 10 Center Drive, Building \\ 10, Bethesda, MD 20892-0001, USA; Department of \\ Medicine, Division of Endocrinology and Metabolism, \\ Georgetown University, Washington D.C, USA
}

Received: March 24, 2021; Accepted: April 09, 2021; Published: April 16, 2021

\section{Editorial}

For over 30 years, our laboratory has dedicated research efforts toward improving the accuracy of diagnostic testing. Our research program originates from key interactions with the former head of endocrinology and thyroid expert at Children's National Medical Center, Dr. Wellington Hung. Dr. Hung called attention to a critical problem occurring in thyroid function testing: the results for Free Thyroxine (FT4) measured by Immunoassay (IA) often did not agree with values obtained for Thyrotropin Stimulating Hormone (TSH) nor with the patient's clinical condition, especially in cases where TSH was elevated. In other words, FT4 testing by IA was frequently disagreeing with the clinical diagnosis possibly resulting in inappropriate treatment of hypothyroidism in children. Therefore, accurate diagnostic testing is paramount.

There are many factors that need to be considered when evaluating the accuracy of diagnostic tests. The most obvious concern has to do with the specificity of the testing mechanism employed. While direct IA measurements for small molecule analytes are convenient, relatively economical, and precise [1], they are also prone to crossreactivity and competitive binding interactions which can lead to inaccurate results $[2,3]$. Cross-reactivity has been well-characterized in steroid hormone measurements, where antibodies are unable to differentiate among numerous biologically distinct hormones with a shared molecular scaffold [4]. Competitive binding interactions, are often alluded to in the literature, but rarely addressed directly $[5,6]$. Recently, our laboratory found that naturally occurring abnormal fluctuations of specific binding proteins (Thyroxine Binding Globulin (TBG), Corticosteroid Binding Globulin (CBG)) affected the accuracy of total and free Triiodothyronine (TT3 and FT3) and cortisol measurements by IA $[7,8]$. By contrast, measurements of FT4, FT3, TT3 and cortisol by Liquid Chromatography Tandem Mass Spectrometry (LC-MS/MS) correlate accurately with the clinical presentation [8-12]. Assays by LC-MS/MS typically require a protein precipitation, or purification step (dialysis, ultrafiltration) during sample preparation which physically removes competing binding proteins from the sample. Additionally, analyte identification and quantification is determined by the detection of specific masses resulting from unique fragmentation patterns, rather than binding affinity. Thus, LC-MS/MS methods have greater specificity than their IA counterparts, and should be the preferred method for evaluating thyroid and adrenal function among other important steroid profiles.

Another important, yet often overlooked, factor to consider when assessing diagnostic results is whether the reference interval provided is appropriate. A recently published study [13] highlights the importance of precision medicine in the definition of pediatric thyroid function test reference intervals, and brings forth necessary discussion of key issues surrounding the measurement of and diagnostic reliance on TSH that we believe may benefit the readership. In addition to the variables described by Oron et al. that affect TSH levels [14], steroid concentration must also be taken into consideration for the accurate reporting of TSH in thyroid function evaluation [10,11,14-18]. This effect was clearly demonstrated by our previous investigation in which we observed substantial decreases in TSH measurements after ACTH stimulation, when steroid concentrations are high [11]. It is important to remember that neonatal steroid hormone levels are much higher than those in adults, as newborns at birth have similar concentrations of estradiol and progesterone to their mothers [19]. Neonatal screening programs usually occur between days 2-5 postpartum. Higher steroid hormone levels would lower TSH serum levels in newborns, potentially leading to falsely normal values of $\mathrm{TSH}$, masking an underactive thyroid. This scenario is particularly concerning as undiagnosed hypothyroidism in newborns can lead to serious permanent deficiencies in neurocognitive development [20]. While the incidence of congenital hypothyroidism may be considered rare (1 in 3000 births) [21], the long-term effects of a missed diagnosis are of great cost to the patient. At present the measurement of TSH is the primary screening strategy used to detect and diagnose congenital hypothyroidism in newborns $[22,23]$. This recommendation needs to be revisited given the common variation in serum TSH levels and often inaccurate reference intervals.

Many reference intervals for TSH are not accurate for the populations they serve-whether composed of different ethnicities or of patients with distinct steroid profiles. Hypothyroidism affects a significant population of patients, yet screening and diagnosis often rely heavily on TSH concentrations, which are variable, especially depending the concentration of steroid hormones present. Steroid hormone concentrations are clearly affected by age (over puberty and adolescence) and sex, and also by very commonly prescribed medications such as statins [24], oral contraceptives [18] and drugs 
to alleviate seasonal allergies [25]. For the past three decades we have implemented IA test reflexing for when measurements of TSH are either high or low, automatically sending samples for thyroid hormone measurements by Liquid Chromatography Tandem Mass Spectroscopy (LC-MS/MS). In our experience, TSH levels that are lower than $1 \mathrm{mIU} / \mathrm{L}$ typically correspond to patients who are taking a regimen of steroids. We suggest that thyroid function assessments take into account more than just TSH. Experience has shown that IA's for TT3 correlate well with measurements of TT3 by LC-MS/ MS and with the clinical picture at normal to high concentrations $[10,11,26,27]$. At low TT3 concentrations, however, this is not true. IA shows a positive bias of 30-40 ng/dl [28]. Of course, our advice for relying on these additional diagnostic markers requires that the appropriate reference intervals [29] also be established.

\section{Funding}

Relevant research on TSH and thyroid hormone measurement and reference intervals by the Soldin Laboratory referenced in this letter is funded by Dr. Soldin's NIH Intramural Research Award.

\section{Author Contribution(s)}

Anastasia Gant Kanegusuku: Conceptualization; writingoriginal draft preparation; writing-review and editing.

Steven J. Soldin: Conceptualization; resources; writing-original draft preparation; writing-review and editing.

\section{References}

1. Cali JP. An idea whose time has come. Clin Chem. 1973; 19: 291-293.

2. Hoofnagle $\mathrm{AN}$, Wener $\mathrm{MH}$. The fundamental flaws of immunoassay and potential solutions using tandem mass spectrometry. J Immunol Methods. 2009; 347: 3-11.

3. Taylor AE, Keevil B, Huhtaniemi IT. Mass spectrometry and immunoassay how to measure steroid hormones today and tomorrow. Eur J Endocrinol. 2015; 173: D1-D12

4. Handelsman DJ, Wartofsky L. Requirement for mass spectrometry sex steroid assays in the journal of clinical endocrinology and metabolism. J Clin Endocrinol Metab. 2013; 98: 3971-3973.

5. van Deventer H, Mendu DR, Remaley AT, Soldin SJ. Inverse log-linear relationship between thyroid- stimulating hormone and free thyroxine measured by direct analog immunoassay and tandem mass spectrometry. Clin Chem. 2011; 57: 122-127.

6. Wang R, Nelson JC, Weiss RN, Wilcox RB. Accuracy of free thyroxine measurements across natural ranges of thyroxine binding to serum proteins. Thyroid. 2000; 10: 31-39.

7. Haq N, Araque KA, Gant Kanegusuku AL. Are serum cortisol measurements by immunoassays reliable: A case series? Med Res Arch. 2020; 8.

8. Gant Kanegusuku AL, Araque KA, Nguyen H, Wei B, Hosseini S, Soldin SJ The effect of specific binding proteins on immunoassay measurements of total and free thyroid hormones and cortisol. Ther Adv Endocrinol Metab. 2021; $12: 1-9$.

9. Sheikh SI, Parikh TP, Kushchayeva Y. TSH should not be used as a Single Marker of Thyroid Function. Annals Thyroid Res. 2018; 4: 151-154.

10. Ling C, Sun Q, Khang J. Does TSH Reliably Detect Hypothyroid Patients? Annals Thyroid Res. 2018; 4: 122-125.
11. Jonklaas J, Kahric-Janicic N, Soldin OP. Correlations of free thyroid hormones measured by tandem mass spectrometry and immunoassay with thyroid-stimulating hormone across 4 patient populations. Clin Chem. 2009; 55: $1380-1388$

12. Welsh KJ, Soldin SJ. Diagnosis of Endocrine Disease: How Reliable Are Free Thyroid and Total T3 Hormone Assays? Eur J Endocrinol. 2016; 175: R255-R263.

13. Oron T, Lazar L, Feldhamer I, Manor M, Koren-Morag N, Tenenbaum A, et al. Pediatric reference values of TSH should be personalized according to BMI and ethnicity. Eur J Endocrinol. 2020; 183: 419-426.

14. Haugen BR. Drugs that suppress TSH or cause central hypothyroidism. Best Pract Res Clin Endocrinol Metab. 2009; 23: 793-800

15. Wilber JF, Utiger RD. The effect of glucocorticoids on thyrotropin secretion. $J$ Clin Investig. 1969; 48: 2096-2103.

16. Brabant A, Brabant G, Schuermeyer T. The role of glucocorticoids in the regulation of thyrotropin. Acta Endocrinol. 1989; 121: 95-100.

17. Jensen J, Nolan G, Jubiz W. The effect of prednisone on serum thyrotropin thyroxine and triiodothyronine concentrations in hypothyroid patients. J Clin Endocrinol Investig. 1978; 1: 171-173.

18. Benvenga S, Di Bari F, Granese R, et al. Circulating thyrotropin is upregulated by estradiol. J Clin Transl Endocrinol. 2018; 11: 11-17.

19. Soldin SJ, Wong EC, Brugnara C, Soldin OP, editors. Pediatric reference intervals. $7^{\text {th }}$ edition. Washington, DC: AACC Press. 2011.

20. Selva KA, Harper A, Downs A. Neurodevelopmental outcomes in congenital hypothyroidism: comparison of initial T4 dose and time to reach target T4 and TSH. J Pediatr. 2005; 147: 775-780.

21. Grosse SD, Van Vliet G. Prevention of intellectual disability through screening for congenital hypothyroidism: how much and at what level. Arch Dis Child. 2011; 96: 374-379.

22. Rose SM, Brown RS. Update of Newborn Screening and Therapy for Congenital Hypothyroidism. Pediatrics. 2006; 117: 2290-2303.

23. Jacob JJ. Neonatal Screening for Congenital Hypothyroidism with Focus on Developing an Indain Screening Programme. Eur Endocrinol. 2016; 12: 99 103.

24. London E, Tatsi C, Soldin SJ. Acute Statin Administration Reduces Levels of Steroid Hormone Precursors. Horm Metab Res. 2020; 10: 742-746.

25. Williams DM. Clinical Pharmacology of Corticosteroids. Respir Care. 2018 63: 655-670.

26. Jonklaas J, Kahric-Janicic N, Soldin OP, et al. Correlations of free thyroid hormones measured by tandem mass spectrometry and immunoassay with thyroid-stimulating hormone across 4 patient populations. Clin Chem. 2009; 55: 1380-1388.

27. Welsh KJ, Soldin SJ. Diagnosis of Endocrine Disease: How Reliable Are Free Thyroid and Total T3 Hormone Assays? Eur J Endocrinol. 2016; 175 R255-R263.

28. Masika LS, Zhao Z, Soldin SJ. Is measurement of TT3 by immunoassay reliable at low concentrations? A comparison of the Roche Cobas 6000 vs. LC-MSMS. Clin Biochem. 2016; 49: 846-849.

29. Sun $Q$, Avallone L, Stolze B. Demonstration of reciprocal diurnal variation in human serum T3 and rT3 concentration demonstrated by mass spectrometric analysis and establishment of thyroid hormone reference intervals. Ther Adv Endocrinol and Metab. 2020; 11: 1-7. 